\title{
Plasma Protein Biomarkers Correlated with the Development of Diet-Induced Type 2 Diabetes in Mice
}

\author{
Shigeru Okada • Edward O. List • Sudha Sankaran • \\ John J. Kopchick
}

Published online: 6 February 2010

(C) Springer Science+Business Media, LLC 2010

\begin{abstract}
Introduction Early detection, assessment of disease progression, and application of an appropriate therapeutic intervention are all important for the care of patients with type 2 diabetes. Currently, however, there is no simple test for early detection of type 2 diabetes. Established diagnostic tests for the disease including oral glucose tolerance, fasting blood glucose, and hemoglobin A1c are relatively late markers where the disease has already progressed. Since blood is in direct contact with many tissues, we hypothesized that pathological tissue changes are likely to be reflected in proteomic profiles of plasma.

Methods Mice were reared either on regular chow or a high-fat diet at weaning and several physiological responses (i.e., weight, fasting plasma glucose and insulin, and glucose tolerance) were monitored at regular time intervals. Plasma was collected at regular intervals for proteomic analysis by two-dimensional gel electrophoresis and subsequent mass spectrometry.
\end{abstract}

S. Okada E. O. List $\cdot$ S. Sankaran · J. J. Kopchick $(\bowtie)$

Edison Biotechnology Institute, Konneker Research Laboratories,

Ohio University,

The Ridges, Bldg. 25,

Athens, OH 45701-2979, USA

e-mail: kopchick@ohio.edu

S. Okada

Department of Pediatrics, College of Osteopathic Medicine,

Ohio University,

Athens, OH, USA

J. J. Kopchick

Department of Biomedical Science,

College of Osteopathic Medicine, Ohio University,

Athens, $\mathrm{OH}$, USA
Results Onset of hyperinsulinemia with corresponding glucose intolerance was observed in 2 weeks and fasting blood glucose levels rose significantly after 4 weeks on the high-fat diet. Many proteins were found to exist in multiple forms (isoforms). Levels of some isoforms including plasma retinol binding protein, transthyretin, Apolipoprotein A1, and kininogen showed significant changes as early as 4 weeks which coincided with the very early development of glucose intolerance.

Conclusions These results show that a proteomic approach to study the development of type 2 diabetes may uncover unknown early post-translationally modified diagnostic and/or therapeutic protein targets.

Keywords Diabetes $\cdot$ Biomarkers $\cdot$ Mice $\cdot$ Protein isoforms Two-dimensional gel electrophoresis $\cdot$ Plasma

\section{Introduction}

Obesity is a global epidemic [1]. Currently, approximately $33.3 \%$ of males and $35.5 \%$ of females in the United States are obese and the ratio continues to rise [2-4]. Obesity often results in premature morbidity and mortality and is associated with the development of type 2 diabetes $[5,6]$. Recent studies indicate that over $50 \%$ of the type 2 diabetics in the United States are obese [2, 3]. According to recent estimates by the American Diabetes Association, type 2 diabetes mellitus is predicted to affect 300 million worldwide by 2025 [1]. Obesity can precipitate type 2 diabetes, in part, due to an increase in insulin resistance [7]. Despite years of study, the relationship between these conditions and the progression from obesity to type 2 diabetes remains poorly understood. A better understanding of what triggers the onset of diabetes in association with 
obesity and what physiological changes are associated with type 2 diabetes are needed. These types of data will likely lead to better prevention, detection, and disease management schemes with a concomitant reduction in the severity of diabetic complications.

In humans, type 2 diabetes often involves a progression from a normal phenotype, to an obese insulin resistant/hyperinsulinemic state, to frank diabetes [8]. Although the pathogenesis of type 2 diabetes is not completely understood, these stages can be mimicked in C57BL/6J mice fed a high-fat diet. These mice become obese and develop hyperinsulinemia and hyperglycemia [9-12]. The progression from obesity to diabetes in these mice also grows worse with time and with increasing obesity [13]. Thus, the development of obesity, insulin resistance, and hyperglycemia in the C57BL/6J mouse follows the same sequence of the progression of obesity to diabetes in humans and has been used as a disease model.

Considering the current global increase in obesity, metabolic syndrome, and diabetes, new biomarkers would be beneficial since these conditions are either preventable or often reversible with early intervention [14]. However, there is no simple test for early detection of type 2 diabetes. Body mass index or body girth measurements are indices often employed as predictors for the risk factors for type 2 diabetes, however, they are not based on the strict physiological relationships; rather they are correlation based [15]. Established diagnostic tests for diabetes include oral glucose tolerance, fasting blood glucose, and hemoglobin A1c all of which are relatively late markers for the disease [16]. A deeper understanding of the disease pathology is needed, in particular, the early events associated with the obese/hyperinsulinemic state. Since blood is in direct contact with all tissues, pathological tissue changes are likely to be reflected in proteomic profiles of plasma [17]. Plasma samples are readily available; thus, quantitative changes in the plasma proteome could serve as biomarkers indicating the progression toward type 2 diabetes and form the basis of noninvasive diagnostic tests or may even result in therapeutic targets [17].

In this study, we focused on relatively abundant plasma proteins which can be quantified to the nanogram range using two-dimensional gel electrophoresis (2DE) with fluorescent staining and subsequent identification by mass spectrometry (MS). This procedure enabled us to monitor more than 300 proteins during the development of type 2 diabetes using the above mentioned mouse model. We have found isoform-specific changes in several proteins which may serve as biomarkers for a pre-diabetic state as well as clues to the biochemical changes occurring during the development and progression of type 2 diabetes.

\section{Research Design and Methods}

\section{Animals and Diets}

Three-week-old male $\mathrm{C} 57 \mathrm{BL} / 6 \mathrm{~J}$ mice were purchased from Jackson Laboratory (Bar Harbor, ME, USA). Obese and type 2 diabetic mice $(n=20)$ were generated by feeding the animals a high-fat diet (\#F1850, Bioserve, Frenchtown, NJ, USA) in which $17 \%, 27 \%$, and $56 \%$ of the calories were provided by protein, carbohydrates, and fat, respectively $[10-12,18]$. Control mice $(n=10)$ were fed a standard chow (Prolab RMH 3000, PMI Nutrition International, Inc., St. Louis, MO, USA) in which $26 \%$, $60 \%$, and $14 \%$ of the calories were provided by protein, carbohydrates, and fat, respectively. Mice were housed two per cage in a temperature controlled room $\left(22^{\circ} \mathrm{C}\right)$ on a $14 \mathrm{~h}$ light $/ 10 \mathrm{~h}$ dark cycle. All mice were fed ad libitum with free access to water. Animal protocols were approved by Ohio University's Institutional Animal Care and Use Committee.

\section{Plasma Collection, Fasting Blood Glucose, Glucose} Tolerance, and Insulin Measurements

Mice were fasted for $4 \mathrm{~h}$ before blood glucose levels were measured in a drop of tail blood using the ONE TOUCH glucometer from Lifescan (Milpitas, CA, USA). All measurements were taken between 2:00 and 4:00 p.m. to ensure that the values were not impacted by diurnal fluctuations in blood glucose levels. Following these measurements, approximately $200 \mu \mathrm{L}$ of blood was collected in heparinized capillary tubes and plasma was separated by centrifugation at $7,000 \times \mathrm{g}$ for $10 \mathrm{~min}$ at $4^{\circ} \mathrm{C}$ and stored at $-80^{\circ} \mathrm{C}$. A part of the plasma samples was used to quantify insulin levels using an ultrasensitive rat/mouse insulin ELISA kit (ALPCO, Windham, NH, USA). Glucose tolerance was measured by intraperitoneal injection of a $25 \%$ glucose solution at $0.01 \mathrm{~mL} / \mathrm{g}$ body weight after $4 \mathrm{~h}$ of fasting. Blood glucose was measured just prior to $(0 \mathrm{~min})$ and at 30,60, and $90 \mathrm{~min}$ after glucose injection by tail bleeding. In order to double the number of blood collections while minimizing the stress of bleeding, mice were divided into two groups and blood was collected every other week from each group. In this scenario, group A was bled the first week and group B the second.

\section{Two-Dimensional Gel Electrophoresis}

Plasma protein samples stored at $-80^{\circ} \mathrm{C}$ were thawed on ice and protein concentration was determined. Plasma proteins $(250 \mu \mathrm{g})$ were mixed with $300 \mu \mathrm{L}$ of isoelectric focusing (IEF) buffer containing $8 \mathrm{M}$ urea, $1.8 \mathrm{M}$ thiourea, $4 \%$ zwitterionic detergent (CHAPS), and a protease inhibitor 
cocktail (Aldrich Chem. Co., Milwaukee, WI, USA). Tributylphosphine $(15 \mu \mathrm{L} / \mathrm{mL})$ carrier ampholytes and tris (hydroxymethyl) aminomethane buffer (Tris, $1 \mathrm{M}, \mathrm{pH}=8.8$, $20 \mu \mathrm{L} / \mathrm{mL}$ ) were added and incubated for $2 \mathrm{~h}$ at room temperature. The proteins' reduced sulfhydryl groups were then blocked by quickly mixing with iodoacetamide $(120 \mathrm{mM})$. Protein samples $(250 \mu \mathrm{g})$ were added to the immobilized $\mathrm{pH}$ gradient gel strips $(17 \mathrm{~cm}$, pI range of 310, linear gradient, Bio-Rad) and actively rehydrated $\left(50 \mathrm{~mA}\right.$ ) for $16 \mathrm{~h}$ at $20^{\circ} \mathrm{C}$ prior to IEF at $60 \mathrm{kV} / \mathrm{h}$ (Protean IEF cell, Bio-Rad). The voltage was linearly increased from 0 to $6,000 \mathrm{~V}$ during the first $10 \mathrm{~h}$ followed by $10 \mathrm{~h}$ at $6,000 \mathrm{~V}$ with a current limit of $50 \mu \mathrm{A}$ per strip. Following IEF, the middle section of the strips corresponding to a $\mathrm{pI}$ range of approximately 5 to 8 was excised as described by List et al. [12] and equilibrated in a freshly prepared buffer containing $6 \mathrm{M}$ urea, $2 \%(w / v)$ sodium dodecyl sulfate (SDS), $0.375 \mathrm{M}$ Tris/HCl (pH 8.8), 20\% ( $v / v$ ) glycerol for $15 \mathrm{~min}$. The second dimension SDS polyacrylamide gel electrophoresis (PAGE) was performed as described previously with slight modifications [11]. Proteins were separated in $15 \%$ SDS-PAGE $(8 \times 7 \mathrm{~cm})$ at $50 \mathrm{~mA} / \mathrm{gel}$ with a maximum voltage of $300 \mathrm{~V}$ for $2 \mathrm{~h}$ at room temperature. After electrophoresis, the gels were fixed overnight in a solution containing $40 \% \mathrm{EtOH}, 2 \%$ acetic acid, $0.0005 \%$ SDS followed by washing three times in $2 \%$ acetic acid with $0.0005 \%$ SDS and stained using SYPRO Orange (Molecular Probes, Eugene, OR, USA) at 1/5,000 dilution in the washing buffer as described previously $[10-12,18]$.

\section{Quantitative Analysis of Gel Images}

Gel images were captured using a laser-scanner (Pharos FX plus, Bio-Rad) with the excitation-emission setting at 473$520 \mathrm{~nm}$. Quantity of each protein spot was estimated using the PDQuest 7.0.1 program (Bio-Rad) with its built-in model called "Total Density in Gel Image." This model assumes that the total density of an image (i.e., background density plus spot density) will be relatively consistent from gel to gel. The raw quantity of each spot in a gel was determined by dividing the pixel density of the Gaussian model of each spot by the total intensity value of all the pixels in the image. The average background was subtracted from each spot density using the intrinsic function of PDQuest and was used as a representative concentration value of the spot. To compare plasma proteins at different time points, the gel images were first registered using the software's automatic function and corresponding spot locations matched. Then, the accuracy of the registration (matching) was assessed manually by checking the location of each spot relative to other nearby spots. When the spot matching was ambiguous due to crowding of nearby spots or slight gel image distortion, the match was confirmed by comparing MS spectra for multiple spots suspected in a particular region of the gel. Initial search criteria were set at a twofold difference between control and high-fat fed animals using an average of at least five gel images from five individual animals.

\section{Protein Identification by Mass Spectrometry}

Protein spots of interest were manually excised from the gel, frozen in $40 \mu \mathrm{L}$ of water in 96 well plates, and shipped to the Michigan Proteome Consortium at the University of Michigan. Proteins in the gel plugs were subjected to in-gel digestion with trypsin as follows. All procedures were performed at $37^{\circ} \mathrm{C}$. SYPRO Orange stain was removed by incubating gel plugs twice in $50 \mu \mathrm{L}$ of $100 \mathrm{mM}$ ammonium bicarbonate and $50 \mu \mathrm{L}$ of acetonitrile $(\mathrm{ACN})$ for $10 \mathrm{~min}$. Then the gel plugs were dehydrated by incubating in $50 \mu \mathrm{L}$ of ACN for $5 \mathrm{~min}$, followed by removal of the ACN by aspiration and subsequent incubation for $10 \mathrm{~min}$. Samples were then reduced in $50 \mu \mathrm{L}$ of $10 \mathrm{mM}$ DTT for $30 \mathrm{~min}$ and alkylated in $50 \mu \mathrm{L}$ of iodoacetamide for $20 \mathrm{~min}$. The gel plugs were rinsed in $100 \mu \mathrm{L}$ of ACN for $5 \mathrm{~min}$. Finally, the gel plugs were dehydrated by incubating twice in $50 \mu \mathrm{L}$ of $\mathrm{ACN}$ for $5 \mathrm{~min}$, removing $\mathrm{ACN}$ and further incubating for $5 \mathrm{~min}$. Trypsin $(20 \mu \mathrm{g})$ was dissolved in $200 \mu \mathrm{L}$ of $50 \mathrm{mM}$ acetic acid. To $45 \mu \mathrm{L}$ aliquots of trypsin solution, $360 \mu \mathrm{L}$ of water and $360 \mu \mathrm{L}$ of $100 \mathrm{mM}$ ammonium bicarbonate was added. The final trypsin concentration was $6 \mathrm{ng} / \mu \mathrm{L}$. Trypsin solution $(25 \mu \mathrm{L})$ was added to the each gel plug and incubated for 8 to $12 \mathrm{~h}$. After digestion, hydrophilic peptides were extracted with $30 \mu \mathrm{L}$ of extraction solution ( $1 \%$ formic acid and $2 \% \mathrm{ACN})$ for $30 \mathrm{~min}$, then hydrophobic peptides were extracted with $12 \mu \mathrm{L}$ of extraction solution and $12 \mu \mathrm{L}$ of ACN for $30 \mathrm{~min}$. To $30 \mu \mathrm{L}$ of digest extract in the extraction plate, $5 \mu \mathrm{L}$ of CHCA $(5 \mathrm{mg} / \mathrm{mL}$ in $50 \%$ ACN, $0.1 \%$ TFA, $2 \mathrm{mM}$ ammonium citrate) matrix was added. The samples were dried and $5 \mu \mathrm{L}$ of $50 \%$ ACN $/ 0.1 \%$ TFA was added. The solution $(0.5 \mu \mathrm{L})$ was hand-spotted on a 192-well MALDI target and allowed to dry exposed to the normal atmosphere. The Applied Biosystems 4700 Proteomics Analyzer was used to obtain MS spectra in a Reflector positive ion mode. Peptide masses were acquired for the range between 800 and 3,500 Da. MS spectra were summed from 2,000 laser shots from an Nd-YAG laser operating at $355 \mathrm{~nm}$ and $200 \mathrm{~Hz}$. The signal-to-noise ratio (S/N) criterion was set to 25 or greater. The monoisotopic masses were processed for identification. Prior to peak list generation, MS spectra were calibrated using trypsin autodigestion peaks and smoothed. MS peaks were subsequently queried against the National Center for Biotechnology Information (NCBI) database using the Mascot program v.2.1 (www.matrix science.com) for identification using the following search 
parameters (database: NCBInr; species: all; digest: trypsin; max number missed cleavage: 1; Cys modified by: carbamidomethylation; possible modifications: peptide $\mathrm{N}$ terminal Gln to pyroGlu+oxidation of $\mathrm{M}+$ Protein $\mathrm{N}$ terminus acetylated; protein mass: not specified; peptide mass: monoisotopic; mass tolerance: $50 \mathrm{ppm}$ ). MS/MS spectra were obtained in MS/MS $2 \mathrm{kV}$ positive mode. Spectra were acquired for 6,000 laser shots or until 12 peptide fragment ions reached an $\mathrm{S} / \mathrm{N}$ of 100 , whichever was less. Atmosphere with a pressure of approximately $6 \times 10^{-7}$ Torr and collision energy of $2 \mathrm{kV}$ was used to induce fragmentation of the peptides. A 7-point Gaussian smooth was performed before peak selection. A maximum of 65 peaks were submitted for the search with a minimum $\mathrm{S} / \mathrm{N}$ of ten and a maximum peak density of 50 peaks per 200 Da. The NCBI database was first searched without considering modifications then searched with the same parameters as the MS search criteria described above. A peptide charge state of 1+ was used and parent ion mass accuracy was better than $\pm 50 \mathrm{ppm}$. Fragment ion mass accuracy was better than $\pm 0.5 \mathrm{Da}$. Identification was accepted only when two or more peptide fragments were identified as the same protein with a MS/MS ion search score higher than $65(p<0.05)$.

\section{Statistical Analysis}

All the statistical analysis was performed using the SPSS version 14.0 software (Chicago, IL, USA). Mouse physiological data were analyzed by univariate analysis of variance (ANOVA) and presented as the mean \pm SEM. Protein concentration was obtained as a pixel density of a Gaussian model of each spot after average background subtraction. For comparison between control and high-fat fed mice, corresponding spots from at least five gel images obtained from five mice were analyzed by univariate ANOVA. Differences were considered significant at $p<0.05$.

\section{Results}

The average mouse body weights were significantly higher in the high-fat fed group as early as 4 weeks on the diet and thereafter [10-12] (Fig. 1). Fasting blood glucose was consistently and significantly higher after 8 weeks (Fig. 2), and plasma insulin levels were significantly higher in the high-fat fed group after 2 weeks on the diet and thereafter (Fig. 3). The high-fat fed group showed impaired glucose tolerance at 2 weeks, 6 weeks, and throughout the course of the experiment (Fig. 3).

Approximately 200 samples were submitted for MS/MS analysis and 85 spots were identified with at least two ion scores higher than $65(p<0.05)$. Protein identification details of 16 spots correspond to 16 isoforms of four proteins and are listed in Table 1. Two ion scores used for identification of each protein isoform are shown.

Four "spots" were identified as plasma retinol binding protein (RBP4) with MWs of $\sim 20 \mathrm{kD}$ and pIs between 5.3 and 7.0 (Fig. 4). RBP4 isoforms were numbered 1 through 4 from the most acidic to the most basic (Fig. 5). The total plasma concentration of the four RBP4 isoforms was calculated as the sum of fluorescent emission of all four RBP isoforms. The total amount of RBP4 started to increase at 16 weeks and was highly induced at 20 weeks on the high-fat diet. The most acidic form (isoform 1) of RBP4 steadily decreased beginning at 4 weeks on the highfat fed diet while the level of this isoform was unchanged in

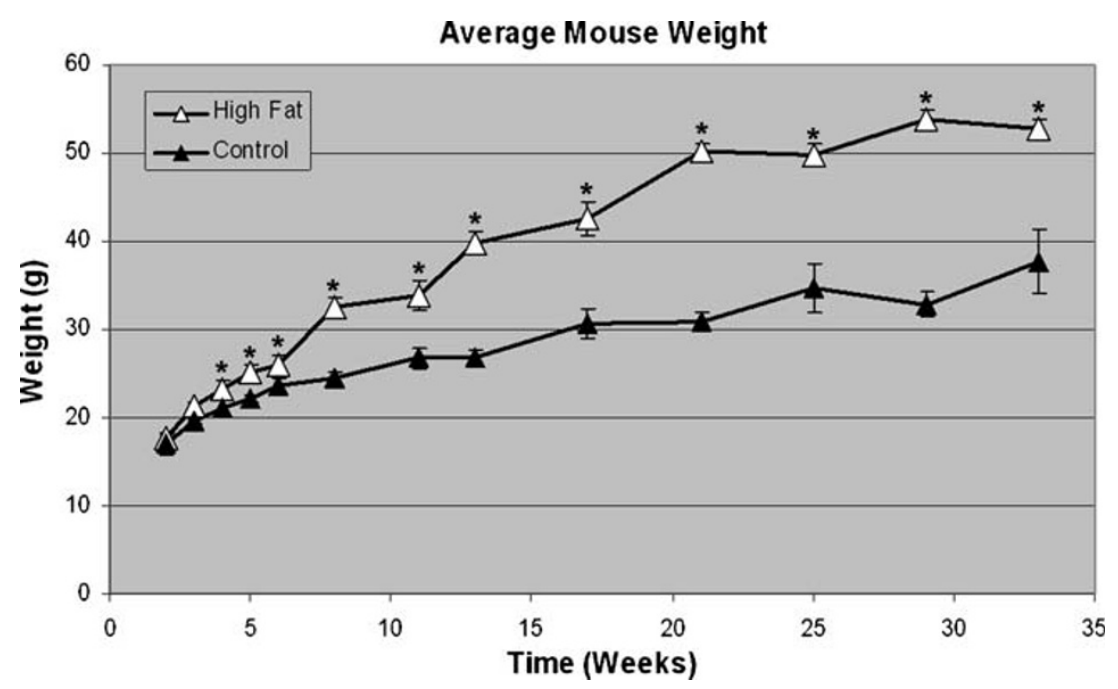

Fig. 1 Effect of high-fat feeding on weight gain. Average weight of control $(n=10)$ and high-fat fed $(n=20)$ mice were plotted for the duration on the high-fat diet. Error bars represent the SEM. Statistical analysis was performed using ANOVA. Single asterisk indicates $p<0.05$ 


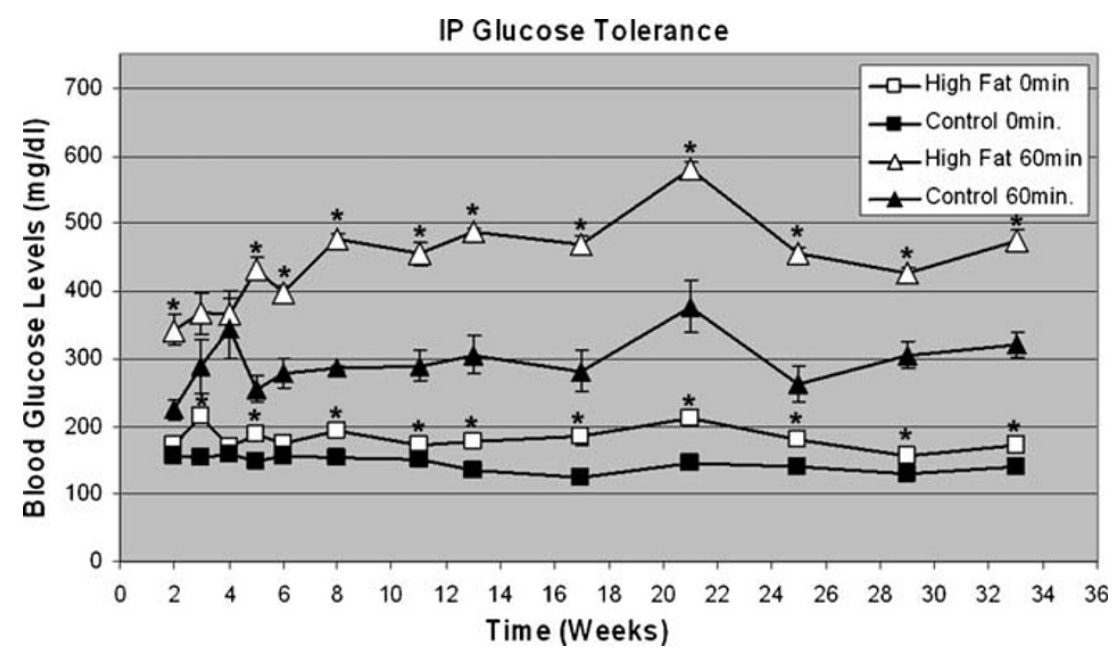

Fig. 2 Effect of high-fat feeding on glucose tolerance. Glucose tolerance was measured by intraperitoneally injecting a $25 \%$ glucose solution at $0.01 \mathrm{~mL} / \mathrm{g}$ body weight after $4 \mathrm{~h}$ of fasting. Blood glucose was measured immediately before the glucose injection $(0 \mathrm{~min})$ and at 30,60 , and $90 \mathrm{~min}$ after the injection by tail bleeding using the ONE TOUCH glucometer from Lifescan (Milpitas, CA, USA). Average blood glucose levels of high-fat fed group $(n=10)$ and control group $(n=5)$ at $60 \mathrm{~min}$ after glucose injection were plotted against time on high-fat

control plasma (Fig. 5, panel 1). Isoform 2 steadily increased in the high-fat fed group with the level significantly higher than controls at 4 weeks on the diet (Fig. 5, panel 2). Isoform 3 (the most abundant RBP4 isoform) did not start to increase until 12 weeks and then sharply rose in the high-fat fed groups (Fig. 5, panel 3). Isoform 4 showed a similar biphasic change in both control and high-fat fed diet. In order to double the number of blood collections while minimizing the stress of bleeding on the mice, each of control $(n=10)$ and high-fat fed $(n=20)$ cohort was divided into two bleeding groups, and blood was collected every other week from each group until 8 weeks. Then blood collection was continued at the indicated time points by alternating between the bleeding groups. Error bars represent the SEM. Statistical analysis was performed using ANOVA. Single asterisk indicates $p<0.05$

mice until 10 weeks. It was significantly induced in the high-fat fed group after 12 weeks (Fig. 5, panel 4).

Six protein spots were identified as transthyretin (TTR) with $\mathrm{MW}$ of $\sim 15 \mathrm{kD}$ and pIs between 5.3 and 6.6. Isoforms were numbered 1 through 6 from the most acidic form to the most basic (Fig. 4). Total plasma concentrations of TTR were not significantly different between the control and

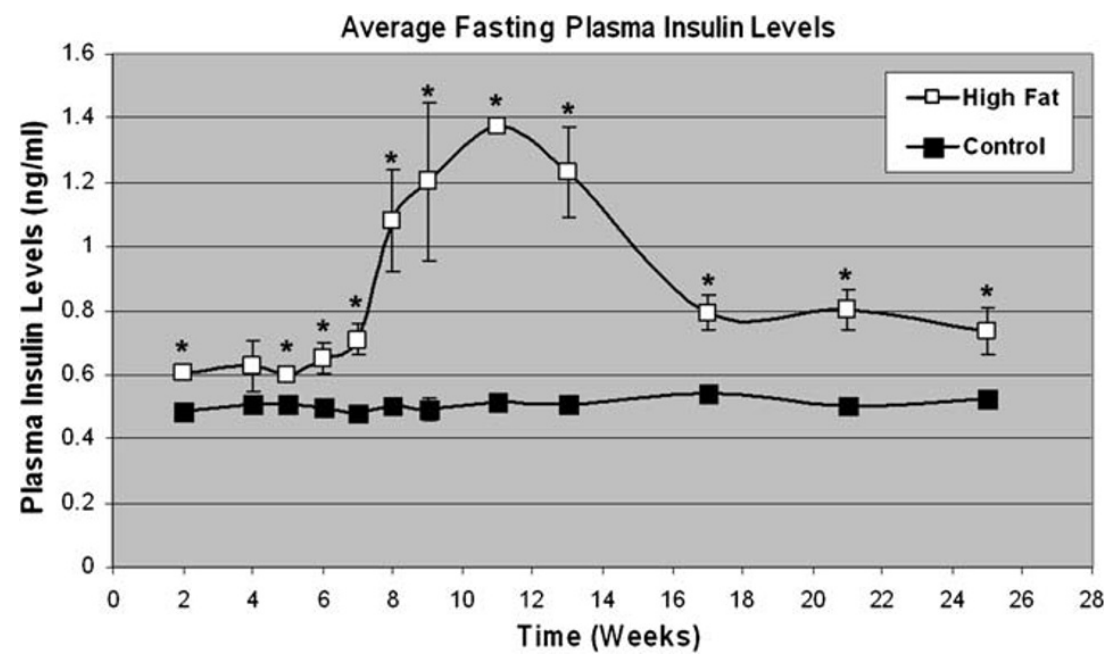

Fig. 3 Effect of high-fat feeding on plasma insulin levels. Average plasma insulin concentrations of control $(n=5)$ and high-fat fed $(n=10)$ mice were plotted for the duration on the high-fat diet. Mice were fasted for $4 \mathrm{~h}$ and approximately $200 \mu \mathrm{L}$ of tail blood was collected in heparinized capillary tubes and plasma was separated by centrifugation at $7,000 \times \mathrm{g}$ for $10 \mathrm{~min}$ at $4^{\circ} \mathrm{C}$ and stored at $-80^{\circ} \mathrm{C}$. A part of the plasma samples was used to quantify insulin levels using an ultrasensitive rat/mouse insulin ELISA kit (ALPCO, Windham, NH, USA). Fasting plasma insulin levels were higher in high-fat fed mice except at 4week time point and the highest level was observed between 8 and 13 weeks. Error bars represent the SEM. Statistical analysis was performed using ANOVA. Single asterisk indicates $p<0.05$ 
Table 1 List of protein identification parameters

\begin{tabular}{|c|c|c|c|c|}
\hline Protein name (isoform number) & Experimental pI & Ion score & Coverage $(\%)$ & Peptide sequence \\
\hline \multirow[t]{2}{*}{$\mathrm{RBP} 4(1)^{\mathrm{a}}$} & \multirow[t]{2}{*}{5.3} & 65 & 4 & K.YWGVASFLQR.G \\
\hline & & 70 & 10 & K.KDPEGLFLQDNIIAEFSVDEK.G \\
\hline \multirow[t]{2}{*}{ RBP 4 (2) } & \multirow[t]{2}{*}{5.5} & 67 & 4 & K.YWGVASFLQR.G \\
\hline & & 72 & 10 & K.KDPEGLFLQDNIIAEFSVDEK.G \\
\hline \multirow[t]{2}{*}{ RBP 4 (3) } & \multirow[t]{2}{*}{5.7} & 66 & 4 & R.FSGLWYAIAK.K \\
\hline & & 74 & 10 & K.YWGVASFLQR.G \\
\hline \multirow[t]{2}{*}{ RBP 4 (4) } & \multirow[t]{2}{*}{7.0} & 71 & 4 & K.YWGVASFLQR.G \\
\hline & & 70 & 10 & K.KDPEGLFLQDNIIAEFSVDEK.G \\
\hline \multirow[t]{2}{*}{ Transthyretin $(1)^{\mathrm{b}}$} & \multirow[t]{2}{*}{5.3} & 66 & 10 & K.TAESGELHGLTTDEK.F \\
\hline & & 89 & 15 & K.TLGISPFHEFADVVFTANDSGHR.H \\
\hline \multirow[t]{2}{*}{ Transthyretin (2) } & \multirow[t]{2}{*}{5.8} & 119 & 15 & K.TLGISPFHEFADVVFTANDSGHR.H \\
\hline & & 65 & 16 & R.HYTIAALLSPYSYSTTAVVSNPQN \\
\hline \multirow[t]{2}{*}{ Transthyretin (3) } & \multirow[t]{2}{*}{6.0} & 127 & 15 & K.TLGISPFHEFADVVFTANDSGHR.H \\
\hline & & 70 & 16 & R.HYTIAALLSPYSYSTTAVVSNPQN \\
\hline \multirow[t]{2}{*}{ Transthyretin (4) } & \multirow[t]{2}{*}{6.3} & 70 & 2 & K.DVFLGTFLYEYSR.R \\
\hline & & 105 & 16 & K.TLGISPFHEFADVVFTANDSGHR.H \\
\hline \multirow[t]{2}{*}{ Transthyretin (5) } & \multirow[t]{2}{*}{6.4} & 157 & 16 & K.TLGISPFHEFADVVFTANDSGHR.H \\
\hline & & 116 & 15 & R.HYTIAALLSPYSYSTTAVVSNPQN \\
\hline \multirow[t]{2}{*}{ Transthyretin (6) } & \multirow[t]{2}{*}{6.6} & 130 & 16 & K.TLGISPFHEFADVVFTANDSGHR.H \\
\hline & & 97 & 15 & R.HYTIAALLSPYSYSTTAVVSNPQN \\
\hline \multirow[t]{2}{*}{ Apolipoprotein A1 (1) ${ }^{\mathrm{c}}$} & \multirow[t]{2}{*}{5.5} & 74 & 3 & K.WKEDVELYR.Q \\
\hline & & 67 & 4 & K.VAPLGAELQESAR.Q \\
\hline \multirow[t]{2}{*}{ Apolipoprotein A1 (2) } & \multirow[t]{2}{*}{5.6} & 74 & 3 & K.WKEDVELYR.Q \\
\hline & & 67 & 4 & K.VAPLGAELQESAR.Q \\
\hline \multirow[t]{2}{*}{ Apolipoprotein A1 (3) } & \multirow[t]{2}{*}{5.7} & 74 & 3 & K.WKEDVELYR.Q \\
\hline & & 88 & 4 & K.VAPLGAELQESAR.Q \\
\hline \multirow[t]{2}{*}{ Apolipoprotein A1 (4) } & \multirow[t]{2}{*}{5.8} & 74 & 3 & K.WKEDVELYR.Q \\
\hline & & 76 & 4 & K.VAPLGAELQESAR.Q \\
\hline Apolipoprotein A1 (5) & 5.9 & 74 & 3 & K.WKEDVELYR.Q \\
\hline & & 76 & 4 & K.VAPLGAELQESAR.Q \\
\hline Apolipoprotein A1 (6) & 6.0 & 74 & 3 & K.WKEDVELYR.Q \\
\hline & & 67 & 4 & K.VAPLGAELQESAR.Q \\
\hline Kininogen $(1)^{\mathrm{d}}$ & 5.5 & 73 & 2 & K.TDGSPTFYSFK.Y \\
\hline & & 90 & 4 & K.EVLGHSIAQLNAENDHPFYYK.I \\
\hline Kininogen (2) & 5.6 & 70 & 2 & K.TDGSPTFYSFK.Y \\
\hline & & 90 & 4 & K.EVLGHSIAQLNAENDHPFYYK.I \\
\hline
\end{tabular}

Two ion scores from MS/MS analysis considered for identification of each protein spot are tabulated above

${ }^{a}$ Protein name: Plasma retinol-binding protein precursor-mouse; Accession code: BAB25881; Nominal mass: 20386; Calculated pI value: 4.98

${ }^{\mathrm{b}}$ Protein name: Transthyretin precursor-mouse; Accession code: VBMS; Nominal mass: 15766; Calculated pI value: 5.77

${ }^{\mathrm{c}}$ Protein name: Apolipoprotein A-I precursor-mouse; Accession code: JC1237; Nominal mass: 30526; Calculated pI value: 5.51

${ }^{\mathrm{d}}$ Protein name: High molecular weight kininogen variant—mouse; Accession code: Q6S9I1_MOUSE; Nominal mass: 53172; Calculated pI value: 4.88

high-fat fed group at most time points (Fig. 6). However, isoform 1 was significantly higher at 6 and 12 weeks in the high-fat fed group (Fig. 6 panel 1). Concentrations of the isoform 2 did not change over time in control mice. However, in the high-fat fed group, the concentration of the isoform 2 was above control levels at 12 weeks and thereafter (Fig. 6, panel 2). Isoform 3 decreased steadily in control plasma and was always higher in the high-fat fed group except at 2 weeks (Fig. 6, panel 3). Plasma concentrations of the isoform 4 were higher in high-fat 


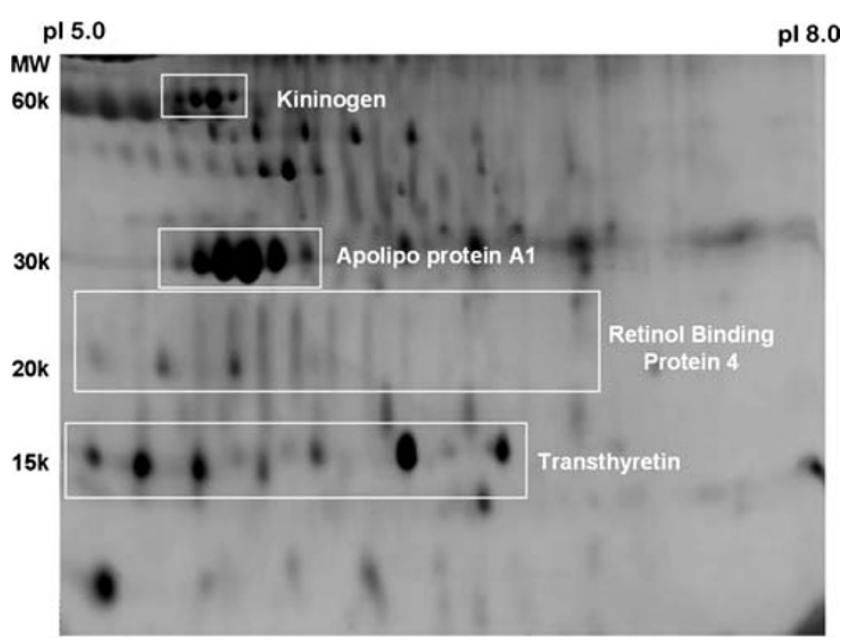

Fig. 4 A typical two-dimensional gel image used in analysis. SYPRO orange-stained two-dimensional gel image of plasma samples taken from standard chow diet control mouse ( 8 weeks) is shown. Approximate $\mathrm{pI}$ range and molecular weight are labeled along the top and left-hand side borders of the gel, respectively. The gel image was captured using a laser-scanner (Pharos FX plus, Bio-Rad) with the excitation-emission setting at $473-520 \mathrm{~nm}$. Each rectangle indicates an area of protein isoform distribution described in Figs. 5, 6, 7, and 8. Spot detection and quantification of each protein spot was performed using the PDQuest 7.0.1 program (Bio-Rad)

fed group except at the two early time points. At 10 weeks, the levels decreased to those found in the control group (Fig. 6, panel 4). The concentration of isoform 5 was constant in controls whereas it showed a steady decline in the high-fat fed group (Fig. 6, panel 5). Concentrations of the isoform 6 were always higher in the high-fat fed group and peaked at 6 weeks while plasma concentrations of this isoform peaked at 8 weeks and declined thereafter in controls (Fig. 6, panel 6).

Six closely associated protein spots were identified as Apolipoprotein A1 (ApoA1). MWs of the six isoforms were $\sim 30 \mathrm{kD}$ and the pIs ranged from $\mathrm{pH} 5.5$ to 6.0. ApoA1 isoforms were numbered 1 through 6 from the most acidic form to the most basic (Fig. 4). The total concentration of ApoA1 was induced in high-fat fed group at 2 weeks and declined with time except at the 12-week point. Plasma concentration of isoform 1 peaked twice in both control and the high-fat fed mice; however, the timing of the two peaks were observed 2 weeks earlier in the high-fat fed group (Fig. 7, panel 1). Isoform 2 was always higher in the controls except at 2 weeks (Fig. 7, panel 2). Isoform 3 was highly induced earlier in high-fat fed group and then decreased to the same levels as controls by 6 weeks and remained low thereafter (Fig. 7, panel 3). This isoform is the most abundant form of ApoA1. Isoform 4 showed a single peak at 6 weeks in controls and was highly induced in the high-fat fed group and returned to control levels at 16 weeks (Fig. 7, panel 4). The concentration of isoform 5 was higher initially in the control relative to the high-fat fed group at 2 weeks. However, it was highly induced in the high-fat fed group at 4 and 6 weeks while levels in the control group were unchanged. Levels of this isoform returned to control levels at 10 weeks (Fig. 7, panel 5). Isoform 6 steadily decreased in the control group while exhibiting two peaks at 8 and 12 weeks in the high-fat fed group (Fig. 7, panel 6).

Two protein spots were identified as kininogen. The MW of these two proteins were $\sim 70 \mathrm{kD}$ and the pIs were approximately 5.5 (Fig. 4). Total concentrations of kininogen were similar between control and high-fat fed groups (Fig. 8). However, the plasma concentration of acidic isoform was highly induced in the high-fat fed group while the concentration remained unchanged in controls (Fig. 8 panel 1). This isoform is also the dominant form of kininogen. The basic isoform was significantly upregulated in the high-fat fed group at the 2-week time point and stayed approximately three to fourfold higher than controls thereafter (Fig. 8, panel 2).

\section{Discussion}

We have performed proteomic analysis between control and high-fat fed obese/diabetic mice and quantified over 300 of the most abundant plasma proteins. One of the major difficulties in studying the plasma proteome is that several proteins, including albumin and immunoglobulins, represent approximately $80 \%$ of total plasma proteins. These proteins give substantial background to the $2 \mathrm{DE}$ profiles and make analysis difficult [17]. To circumvent these problems, we resolved relatively abundant and low molecular weight proteins (below $\sim 80 \mathrm{kD}$ ) using high concentration SDS-PAGE gels and quantified the digitized images following fluorescent staining and laser scanning. An advantage of 2DE compared to other separation methods, such as HPLC, LC-MS/MS, or protein chips, is the quality of protein resolution and amount of data provided [19]. Also, densitometric analyses combined with laser scanning of proteins are highly quantitative. A median coefficient of variation of approximately $8 \%$ and an interquartile range of $8 \%$ to $10 \%(n=5)$ has been reported using similar parameters as used in our procedure (i.e., fluorescent staining, PDQuest software) [20]. We have produced nearly identical gel images even across animals within same treatment group [11, 12].

Identity of each protein spot was determined by MS (MALDI-TOF) followed by MS/MS (TOF-TOF). Accuracy of probability based protein identification is also enhanced by focusing on relatively small proteins. Previous efforts successfully identified biomarkers for cancer and other diseases using these methods [21,22]. We have also found skin biomarkers of diabetes using this approach [11]. Thus, 

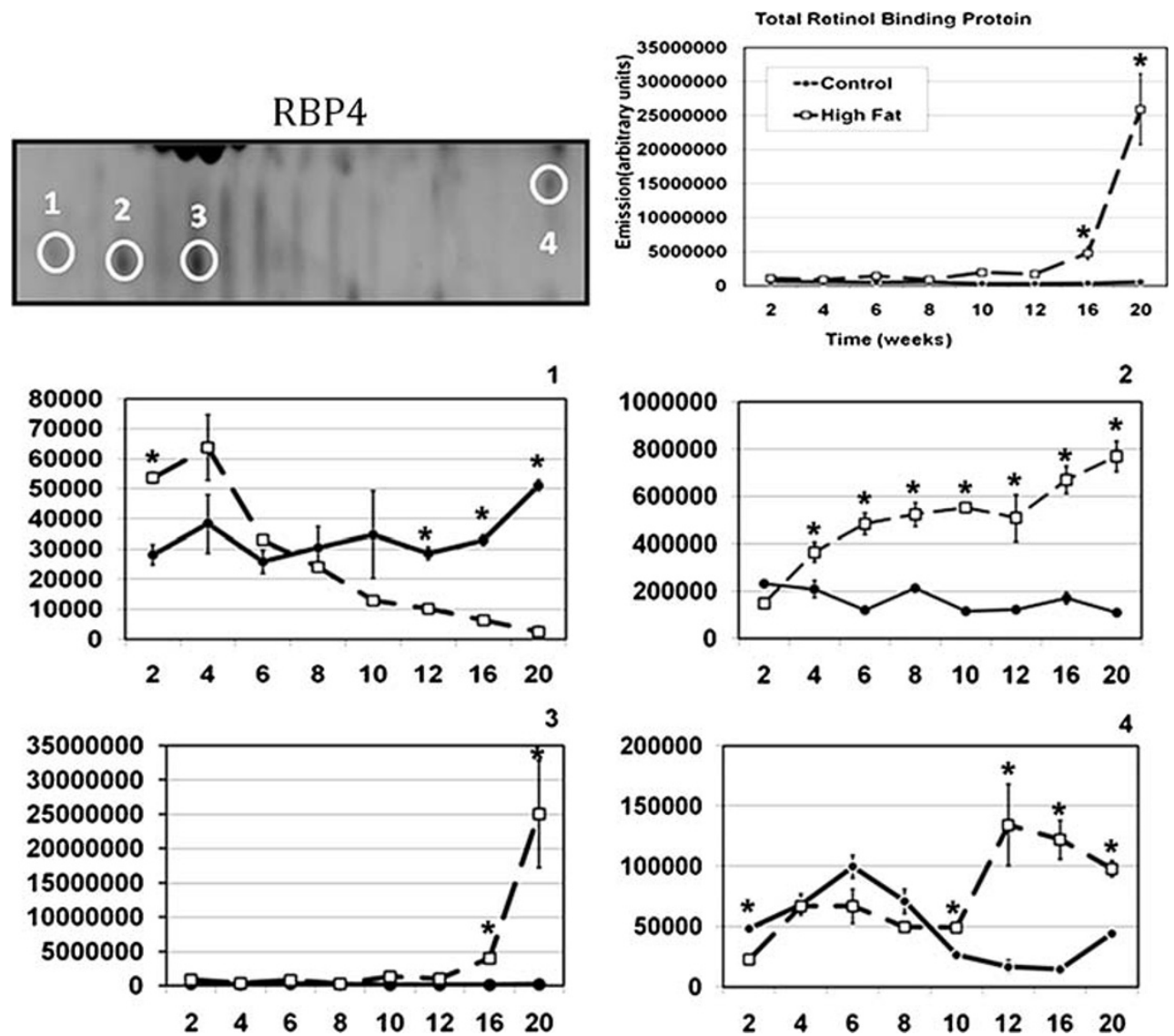

Fig. 5 Quantity changes of RBP4 isoforms. Locations of RBP4 isoforms are indicated by circles in the partial image of twodimensional gel described in Fig. 4 and numbered 1 to 4 from acidic to basic. Average fluorescence intensity of an indicated isoform is plotted for control $(n=5)$ and high-fat fed $(n=8)$ mice. Plasma sample from each mouse was resolved on a two-dimensional gel, and image was captured using a laser-scanner (Pharos FX plus, Bio-Rad) with the excitation-emission setting at $473-520 \mathrm{~nm}$. Detection, matching, and

quantification of each protein spot were performed using the PDQuest 7.0.1 program (Bio-Rad) assuming loading of equal amount of total protein per gel. All of RBP4 spots were cut out from the polyacrylamide gel and analyzed by MS/MS at the Michigan Proteome Consortium. Concentration of total RBP4 (top right panel) was estimated as a sum of fluorescence intensities of all isoforms. Error bars represent the SEM. Statistical analysis was performed using ANOVA. Single asterisk indicates $p<0.05$

2DE has been shown to be a valid method to separate and quantify protein changes [23]. One of the most important aspects of using $2 \mathrm{DE}$ is the capability of resolving many post-translationally modified proteins. As described below, we have identified several isoforms of four proteins.

RBP4 has been established as an adipokine [24]. Also, RBP4 was recently reported as a strong predictor of insulin resistance. It was demonstrated by western blotting that plasma RBP4 levels were increased from two- to 13 -fold in five independent insulin-resistant mouse models [25, 26]. For example, mice on a high-fat diet for 12 weeks showed a 2.8-fold increase in RBP4 level [25]. We observed similar changes in RBP4 in relation to high-fat feeding and the resulting development of type 2 diabetes (Fig. 5). Induction of the total plasma concentration of RBP4 above control levels was evident at 16 weeks on the high-fat diet and the levels increased more than 50 -fold by 20 weeks. Thus, total RBP4 is a rather late marker of type 2 diabetes, which parallels the change in blood glucose levels. Surprisingly, when each of the four individual RBP4 isoforms was quantified, the concentration change in the isoforms was different from that of the total. For example, isoform 1 (most acidic form) was induced very quickly and peaked at 4 weeks on the high-fat diet and steadily decreased while control levels were relatively constant. The total concentration change in RBP4 was most influenced by isoform 3 since it was the most abundant form. Interestingly, isoform 2 responded very quickly to high-fat feeding. Induction of this spot was significant by week 4 which coincided with the onset of insulin resistance and impaired IP glucose tolerance. Thus, induction of this RBP4 isoform was a very early marker of the progression of mice from normal to obese and insulin resistant. Induction of isoform 4 was observed at 10 weeks and after and was rather late in the progression of obesity to diabetes; thus, this isoform would not be of value in the diagnosis of early or pre-diabetes. 

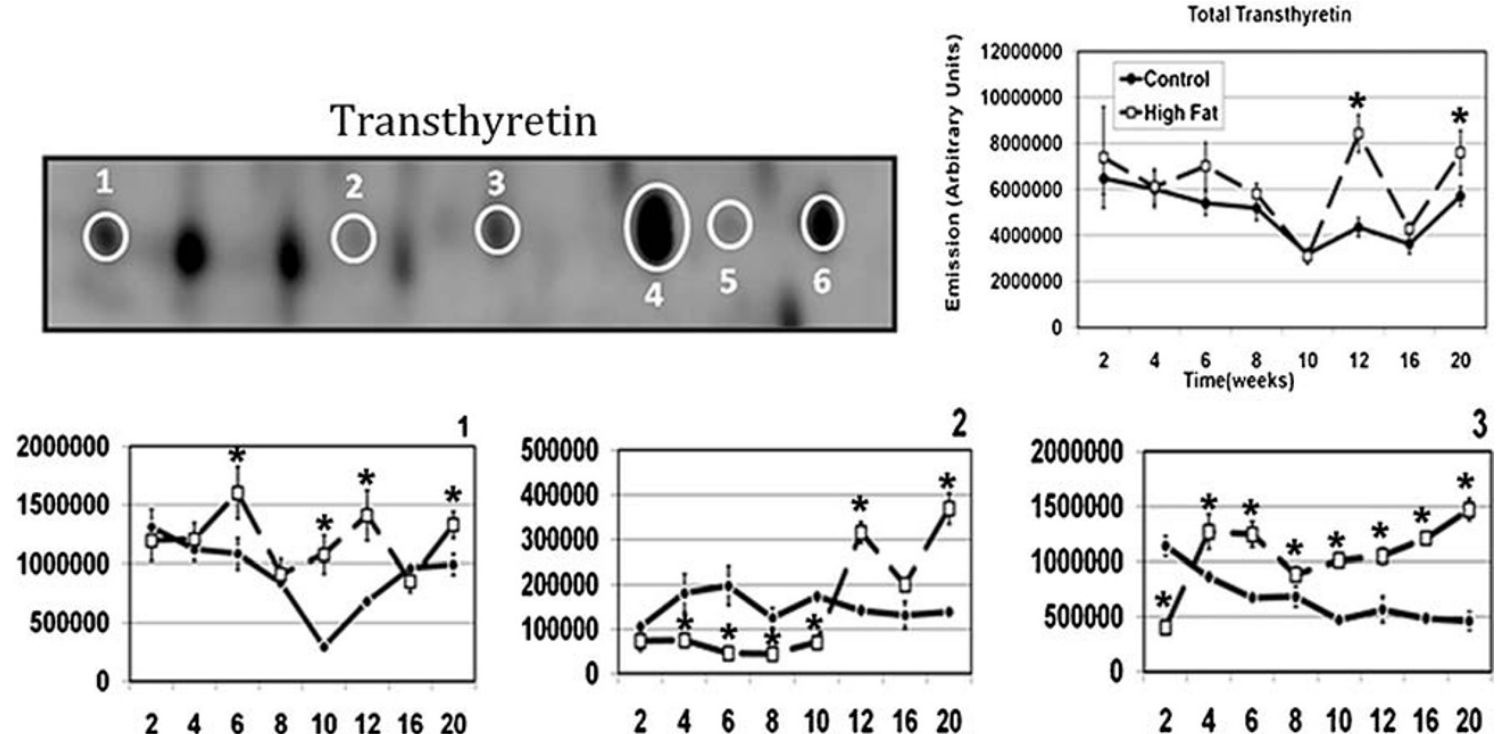

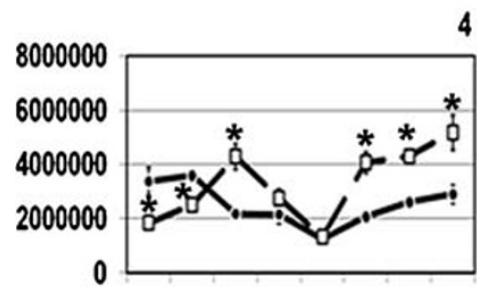

$\begin{array}{lllllllll}2 & 4 & 6 & 8 & 10 & 12 & 16 & 20\end{array}$

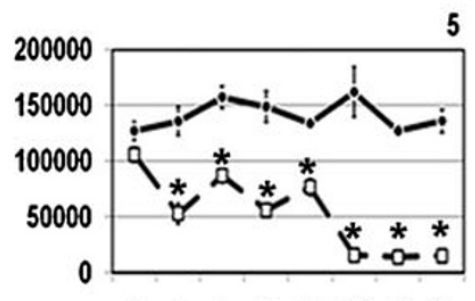

$\begin{array}{llllllll}2 & 4 & 6 & 8 & 10 & 12 & 16 & 20\end{array}$

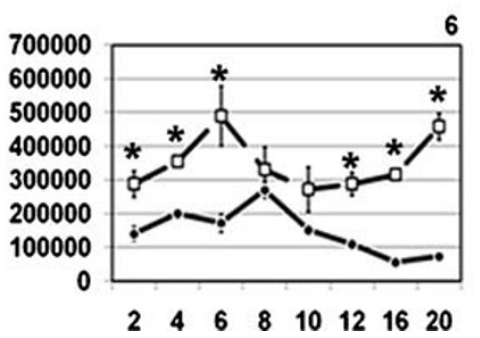

Fig. 6 Quantity changes of transthyretin (TTR) isoforms. Locations of TTR isoforms are indicated by circles in the partial image of twodimensional gel described in Fig. 4 and numbered 1 to 6 from acidic to basic. Average fluorescence intensity of an indicated isoform is plotted for control $(n=5)$ and high-fat fed $(n=8)$ mice. Plasma sample from each mouse was resolved on a two-dimensional gel and image was captured using a laser-scanner (Pharos FX plus, Bio-Rad) with the excitation-emission setting at $473-520 \mathrm{~nm}$. Detection, matching, and

Whether RBP4 could serve as a diagnostic marker or therapeutic target for diabetes in human is still controversial. Circulating RBP4 levels were decreased in patients who showed improved glucose handling after 4 weeks of exercise $(60 \mathrm{~min} /$ day of running and bicycling at least 3 days/week), whereas RBP4 levels did not change in patients who did not improve [26]. However, there was no difference in plasma RBP4 levels when a comparison was made among stratified groups according to BMI of lean, overweight, and obese individuals [27]. These studies did not employ a 2DE approach, thus, the fluctuation in RBP4 isoforms was not established. Obviously, this should (and will) be done.

Although RBP4 is considered to be an adipokine, only $20 \%$ of plasma RBP4 is secreted from adipose tissue while the remainder originates in the liver [24]. We have not assessed the origin of four RBP4 isoforms or the nature of differences among them. These isoforms may arise from different tissue sources and/or changes in protein produc- quantification of each protein spot were performed using the PDQuest 7.0.1 program (Bio-Rad) assuming loading of equal amount of total protein per gel. All of TTR spots were cut out from the polyacrylamide gel and analyzed by MS/MS at the Michigan Proteome Consortium. Concentration of total TTR (top right panel) was estimated as a sum of fluorescence intensities of all isoforms. Error bars represent the SEM. Statistical analysis was performed using ANOVA. Single asterisk indicates $p<0.05$

tion and processing due to tissue specific pathologic processes during the development of diabetes.

RBP4 is a carrier of retinol. A single molecule of RBP4 complexes with homotetrameric TTR in plasma and transports retinol and throxine simultaneously [28]. RBP4 and TTR are $\sim 21$ and $\sim 14 \mathrm{kD}$, respectively, and the complex of $\sim 76 \mathrm{kD}$ is assumed to "escape" glomerular filtration, thus, prolonging their plasma half life [28]. Therefore, it was of interest to determine whether the concentration change in TTR also paralleled that of RBP4. We identified six isoforms of TTR (Fig. 6). Concentration changes of each isoform, as well as the total change, revealed a complex pattern over the course of the experiment. However, we did not observe an increase in TTR to the same extent as seen in total RBP4 (50-fold). If complex formation between RBP4 and TTR is the only mechanism to prolong plasma half life of RBP4, synthesis and secretion of RBP4 into plasma may be much greater in high-fat fed animals to achieve a steady state of 50 -fold in plasma. Alternatively, 

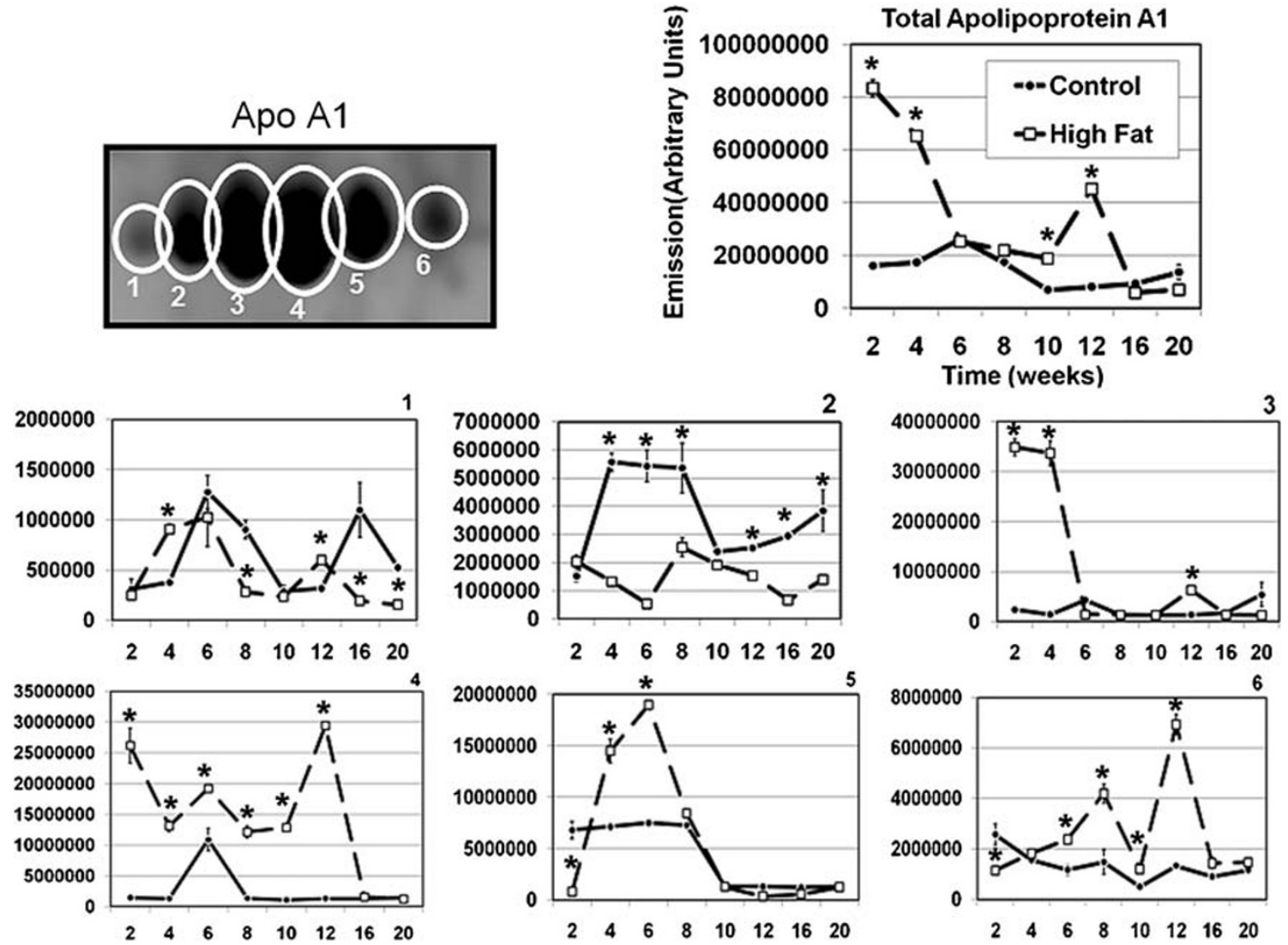

Fig. 7 Quantity changes of Apolipoprotein A-1 (ApoA1) isoforms. Locations of ApoA1 isoforms are indicated by circles in the partial image of two-dimensional gel described in Fig. 4 and numbered 1 to 6 from acidic to basic. Average fluorescence intensity of an indicated isoform is plotted for control $(n=5)$ and high-fat fed $(n=8)$ mice. Plasma sample from each mouse was resolved on a two-dimensional gel and image was captured using a laser-scanner (Pharos FX plus, Bio-Rad) with the excitation-emission setting at 473-520 nm.
Detection, matching, and quantification of each protein spot were performed using the PDQuest 7.0.1 program (Bio-Rad) assuming loading of equal amount of total protein per gel. All of ApoA1 spots were cut out from the polyacrylamide gel and analyzed by MS/MS at the Michigan Proteome Consortium. Concentration of total ApoA1 (top right panel) was estimated as a sum of fluorescence intensities of all isoforms. Error bars represent the SEM. Statistical analysis was performed using ANOVA. Single asterisk indicate $p<0.05$ basal TTR concentrations may be much higher than RBP4 concentrations in plasma.

The total concentration of plasma kininogen was induced after 10 weeks on the high-fat diet while control levels remained relatively constant (Fig. 8 panel 1). Thus, kininogen may be a late marker for type 2 diabetes. We found two isoforms of kininogen with the basic isoform being most abundant (Fig. 8 panel 2). However, we observed induction in the acidic isoform at 4 weeks on the diet and thereafter that was threefold higher than control levels (Fig. 8. panel 3). Thus, this acidic isoform of kininogen may be used as an early marker of an insulin resistant state. Although, the nature of involvement of kininogen with the development of diabetes is not clear, there is evidence that it may play an important role in the process. Deficiency in kininogen in rats leads to enhanced insulin sensitivity [29]. Also, kininogen physically associates with adiponectin, one of the adipokines with anti- diabetic and anti-inflammatory activities [30]. More recently, differential regulation of kinin receptor (B1 and B2) expression in white adipose tissue, hypothalamus, liver, and stomach was demonstrated in ob/ob mice [31].

ApoA1 is the primary constituent of plasma high density lipoprotein (HDL). The protein promotes cholesterol efflux from tissues to the liver for excretion or recycling [32]. Increased levels of HDL have been correlated with a decreased risk of atherosclerosis which is a primary cause of cardiovascular disease [33]. ApoA1 activates lecithin cholesterol acyltransferase which is responsible for the formation of most plasma cholesteryl esters. Absence or specific mutations in ApoA1 has been reported to cause reduction or deficiency of HDL. Also some mutations are associated with dyslipidemia. In addition, ApoA1 bound to HDL has been reported to alter its functions due to various PTMs which include oxidation, nitrosylation, and chlorination [32]. We identified six ApoA1 isoforms. Overall, these 

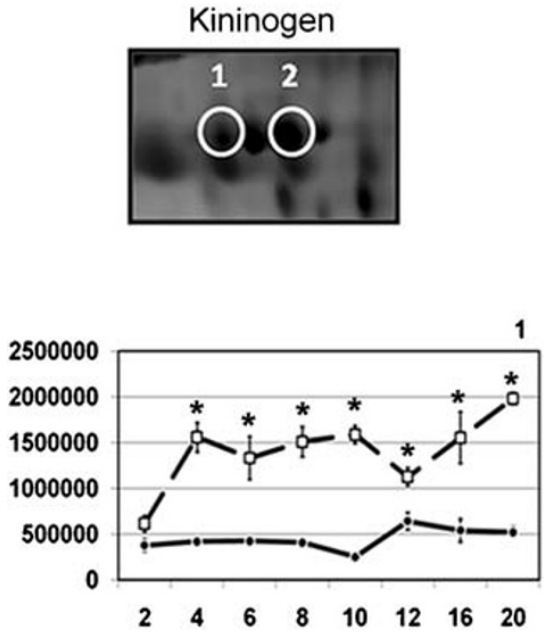

Fig. 8 Quantity changes of kininogen isoforms. Locations of kininogen isoforms are indicated by circles in the partial image of two-dimensional gel described in Fig. 4 and numbered 1 and 2 from acidic to basic. Average fluorescence intensity of an indicated isoform is plotted for control $(n=5)$ and high-fat fed $(n=8)$ mice. Plasma sample from each mouse was resolved on a two-dimensional gel and image was captured using a laser-scanner (Pharos FX plus, Bio-Rad) with the excitation-emission setting at 473-520 nm. Detection,

isoforms are highly induced by 2 weeks on the high-fat diet and then decrease (Fig. 7, total). Isoforms 1, 4, and 6 contribute to the second smaller induction observed around 12 weeks (Fig. 7, panels 1, 4, and 6). Interestingly all isoforms were reduced to basal levels in 20 weeks. Elucidation of the functional difference among these isoforms may contribute to the understanding of early changes of cholesterol metabolism associated with development of type 2 diabetes.

Proteomics has been used as a diagnostic tool to detect changes in the plasma that correlated with ovarian [30] or prostate [31] cancer or with the likelihood of developing hepatocellular carcinoma due to chronic hepatitis B virus infection [32]. However, due to the methodology employed, protein isoforms and/or post-translationally modified versions were not documented. As we have reported here, if the detection method (such as ELISA) of a particular protein biomarker does not distinguish protein isoforms, then the most abundant form of the protein will be reflected in the overall value in the measurement. However, if methods are developed to detected specific protein isoforms, then perhaps new biomarkers of the disease state or pre-disease state may be determined. We realize that interpretation of quantitative proteomic data has to be performed with caution. Many biomarker discoveries were criticized for not being reproducible possibly due to sampling bias [34]. In our mouse model of obesity-
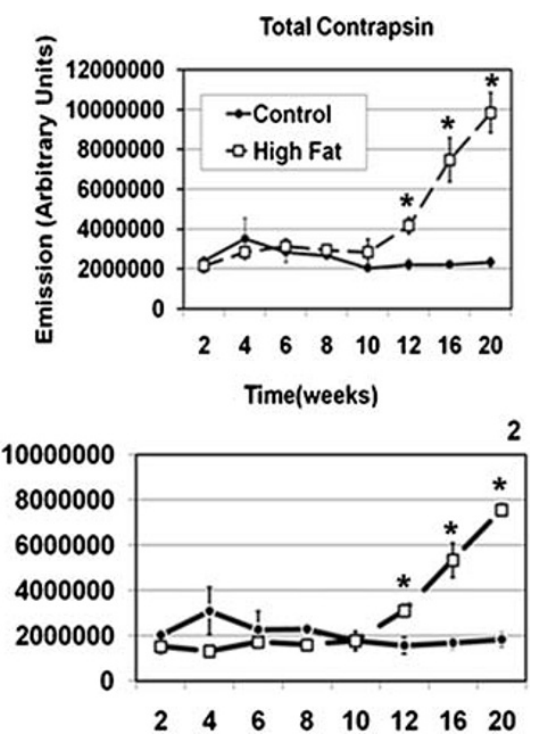

matching, and quantification of each protein spot were performed using the PDQuest 7.0.1 program (Bio-Rad) assuming loading of equal amount of total protein per gel. All of kininogen spots were cut out from the polyacrylamide gel and analyzed by MS/MS at the Michigan Proteome Consortium. Concentration of total TTR (top right panel) was estimated as a sum of fluorescence intensities of all isoforms. Error bars represent the SEM. Statistical analysis was performed using ANOVA. Single asterisk indicates $p<0.05$

induced diabetes, we believe that sampling bias is minimal since the mice are of the same genetic background and are well controlled in terms of diet and upkeep. We also realize that the four proteins reported here have recently been shown to be associated with mouse lung cancer model [35]; however, changes in isoforms of these proteins have not been reported. Investigation of mechanisms associated with these protein concentration changes may reveal common regulatory mechanisms associated with these two seemingly independent diseases. Importantly, we do not know the nature of the modifications of proteins reported in our study and are currently attempting to ascertain these exact posttranslational modifications. Characterization of the chemical nature of these protein isoforms, determination of the tissue that produce them, and the relationship between these isoforms and other interacting proteins-all of these, as they related to the causal events of pre-diabetes and diabetes, may lead to a further understanding of the progression from a pre-diabetic state to that of type 2 diabetes. Also, proteomic profiling as well as extension of these results to humans is of obvious importance.

Acknowledgments JJK is supported by funds from NIA (AG19899), NIDDK (DK075436), the State of Ohio's Eminent Scholar Program that includes a gift from Milton and Lawrence Goll, The Diabetes Research Initiative and The BioMolecular Innovation and Technology Program at Ohio University, and the AMVETS. 


\section{References}

1. Zimmet P, Alberti KG, Shaw J. Global and societal implications of the diabetes epidemic. Nature. 2001;414(6865):782-7.

2. Wickelgren I. Obesity: how big a problem? Science. 1998;280 (5368):1364-7.

3. Kopelman PG. Obesity as a medical problem. Nature. 2000;404 (6778):635-43.

4. Bessesen DH. Update on obesity. J Clin Endocrinol Metab. 2008;93(6):2027-34.

5. Bjorntorp P. Metabolic implications of body fat distribution. Diabetes Care. 1991;14(12):1132-43.

6. Emery EM, Schmid TL, Kahn HS, Filozof PP. A review of the association between abdominal fat distribution, health outcome measures, and modifiable risk factors. Am J Health Promot. 1993;7(5):342-53.

7. Clark MG, Rattigan S, Clark DG. Obesity with insulin resistance: experimental insights. Lancet. 1983;2(8361):1236-40.

8. Ramlo-Halsted BA, Edelman SV. The natural history of type 2 diabetes. Implications for clinical practice. Prim Care. 1999;26 (4):771-89.

9. Surwit RS, Kuhn CM, Cochrane C, McCubbin JA, Feinglos MN. Diet-induced type II diabetes in C57BL/6J mice. Diabetes. 1988;37(9):1163-7.

10. Qiu L, List EO, Kopchick JJ. Differentially expressed proteins in the pancreas of diet-induced diabetic mice. Mol Cell Proteomics. 2005;4(9):1311-8.

11. List EO, Berryman DE, Palmer AJ, et al. Analysis of mouse skin reveals proteins that are altered in a diet-induced diabetic state: a new method for detection of type 2 diabetes. Proteomics. 2007;7 (7):1140-9.

12. List EO, Berryman DE, Palmer AJ, et al. Application of bioinformatics and scalable computing to perform proteomic analysis of stomach tissue from diabetic mice. SCPE. 2007;8(2):173-83.

13. Watson PM, Commins SP, Beiler RJ, Hatcher HC, Gettys TW, Differential regulation of leptin expression and function in $\mathrm{A} / \mathrm{J}$ vs. C57BL/6J mice during diet-induced obesity. Am J Physiol Endocrinol Metabol. 2000;279(2):E356-65.

14. Younis N, Soran H, Farook S. The prevention of type 2 diabetes mellitus: recent advances. QJM. 2004;97(7):451-5.

15. Aronne LJ. Classification of obesity and assessment of obesityrelated health risks. Obes Res. 2002;10 Suppl 2:105S-15.

16. Waugh N, Scotland G, McNamee P, et al. Screening for type 2 diabetes: literature review and economic modelling. Health Technol Assess. 2007;11(17):iii-iv, ix-xi, 1-125.

17. Thadikkaran L, Siegenthaler MA, Crettaz D, Queloz PA, Schneider P, Tissot JD. Recent advances in blood-related proteomics. Proteomics. 2005;5(12):3019-34.

18. Kopchick JJ, List EO, Kohn DT, Keidan GM, Qiu L, Okada S. Perspective: proteomics - see "spots" run. Endocrinology. 2002;143(6):1990-4.
19. Smilansky Z. Automatic registration for images of twodimensional protein gels. Electrophoresis. 2001;22(9):1616-26.

20. Raman B, Cheung A, Marten MR. Quantitative comparison and evaluation of two commercially available, two-dimensional electrophoresis image analysis software packages, Z3 and Melanie. Electrophoresis. 2002;23(14):2194-202.

21. Zhang R, Barker L, Pinchev D, et al. Mining biomarkers in human sera using proteomic tools. Proteomics. 2004;4(1):244-56.

22. Huang CM, Ananthaswamy HN, Barnes S, Ma Y, Kawai M, Elmets CA. Mass spectrometric proteomics profiles of in vivo tumor secretomes: capillary ultrafiltration sampling of regressive tumor masses. Proteomics. 2006;6(22):6107-16.

23. Gorg A, Postel W, Gunther S. The current state of twodimensional electrophoresis with immobilized $\mathrm{pH}$ gradients. Electrophoresis. 1988;9(9):531-46.

24. Tsutsumi C, Okuno M, Tannous L, et al. Retinoids and retinoidbinding protein expression in rat adipocytes. J Biol Chem. 1992;267(3):1805-10.

25. Yang Q, Graham TE, Mody N, et al. Serum retinol binding protein 4 contributes to insulin resistance in obesity and type 2 diabetes. Nature. 2005;436(7049):356-62.

26. Graham TE, Yang Q, Bluher M, et al. Retinol-binding protein 4 and insulin resistance in lean, obese, and diabetic subjects. N Engl J Med. 2006;354(24):2552-63.

27. Janke J, Engeli S, Boschmann M, et al. Retinol-binding protein 4 in human obesity. Diabetes. 2006;55(10):2805-10.

28. Raghu P, Sivakumar B. Interactions amongst plasma retinolbinding protein, transthyretin and their ligands: implications in vitamin A homeostasis and transthyretin amyloidosis. Biochim Biophys Acta. 2004;1703(1):1-9.

29. Damas J, Bourdon V, Lefebvre PJ. Insulin sensitivity, clearance and release in kininogen-deficient rats. Exp Physiol. 1999;84 (3):549-57.

30. Wang Y, Xu LY, Lam KS, Lu G, Cooper GJ, Xu A. Proteomic characterization of human serum proteins associated with the fat-derived hormone adiponectin. Proteomics. 2006;6(13): 3862-70.

31. Abe KC, Mori MA, Pesquero JB. Leptin deficiency leads to the regulation of kinin receptors expression in mice. Regul Pept. 2007;138(2-3):56-8.

32. Zannis VI, Chroni A, Krieger M. Role of apoA-I, ABCA1, LCAT, and SR-BI in the biogenesis of HDL. J Mol Med. 2006;84 (4):276-94.

33. Kapur NK, Ashen D, Blumenthal RS. High density lipoprotein cholesterol: an evolving target of therapy in the management of cardiovascular disease. Vasc Health Risk Manag. 2008;4(1): $39-57$.

34. Ransohoff DF. Promises and limitations of biomarkers. Recent Results Cancer Res. 2009;181:55-9.

35. Chatterji B, Borlak J. A 2-DE MALDI-TOF study to identify disease regulated serum proteins in lung cancer of c-myc transgenic mice. Proteomics. 2009;9(4):1044-56. 